\title{
Report from the National Society of Genetic Counselors Service Delivery Model Task Force: A Proposal to Define Models, Components, and Modes of Referral
}

\author{
Stephanie A. Cohen - Shanna L. Gustafson • \\ Monica L. Marvin • Bronson D. Riley • \\ Wendy R. Uhlmann • S. Bonnie Liebers • \\ Julie A. Rousseau
}

Received: 16 November 2011 / Accepted: 19 April 2012/Published online: 8 May 2012

(C) National Society of Genetic Counselors, Inc. 2012

\begin{abstract}
The Service Delivery Model Task Force (SDMTF) was appointed in 2009 by the leadership of the National Society of Genetic Counselors (NSGC) with a charge to research and assess the capacity of all existing service delivery models to improve access to genetic counseling services in the context of increasing demand for genetic testing and counseling. In approaching this charge, the SDMTF found that there were varying interpretations of what was meant by "service delivery models" and the group held extensive discussions about current practices to arrive at consensus of proposed definitions for current genetic service delivery models, modes of referral and components of service delivery. The major goal of these proposed definitions is to allow for conversations to begin to address the charge to the committee. We propose that current models of service delivery can be defined by: 1) the methods in which genetic counseling services are delivered (In-person, Telephone, Group and Telegenetics), 2) the way
\end{abstract}

S. A. Cohen $(\square)$

Cancer Genetics Risk Assessment Program, St. Vincent Hospital,

8301 Harcourt Rd. \#100,

Indianapolis, IN 46260, USA

e-mail: sacohen@stvincent.org

\section{S. L. Gustafson • W. R. Uhlmann}

Departments of Internal Medicine and Human Genetics, University of Michigan Health System,

300 N Ingalls NI3 A03 SPC 5419,

Ann Arbor, MI 48109, USA

M. L. Marvin

Department of Human Genetics, University of Michigan, 4909 Buhl,

Ann Arbor, MI 48109-5618, USA they are accessed by patients (Traditional referral, Tandem, Triage, Rescue and Self-referral) and 3) the variable components that depend upon multiple factors unique to each service setting. This report by the SDMTF provides a starting point whereby standardized terminology can be used in future studies that assess the effectiveness of these described models to overcome barriers to access to genetic counseling services.

Keywords Service delivery models · Genetic counseling · Access · National Society of Genetic Counselors · Genetics referal

\section{Introduction}

The Service Delivery Model Task Force (SDMTF) was appointed in 2009 by the leadership of the National Society
B. D. Riley

Southeast Nebraska Cancer Center,

201 South 68th Street Place, Suite 200,

Lincoln, NE 68510, USA

\section{S. B. Liebers}

Genetic Counseling Services, Inc.,

1070 Lamplighter Rd.,

Schenectady, NY 12309-1162, USA

URL: www.geneticcounselingservices.com

\section{J. A. Rousseau}

Genetic Diagnostic Laboratory and Department of Laboratory Medicine, Children's Hospital Boston,

9 Hope Avenue,

Waltham, MA 02453, USA 
of Genetic Counselors (NSGC) as part of its 2010-2011 strategic initiatives. The charge of the SDMTF was to research and assess the capacity of all existing service delivery models to improve access to genetic counseling in the context of increasing demand for genetic testing and counseling services.

Traditionally, the focus of medical genetics clinics has been to provide genetic services to patients with rare, Mendelian-inherited conditions. In the early 1990s, there were fewer than 300 genetic tests. By 2011, there were over 2000 clinical genetic tests available (http://www.ncbi.nlm.nih.gov/ sites/GeneTests/). The completion of the Human Genome Project together with advances in our understanding of the genetic basis for common, complex conditions including cancer, heart disease and diabetes, has increased the relevance of genetic services to the general population, thereby leading to increasing numbers of patient referrals. Moreover, advances in genetic testing, most notably the development of lower cost sequencing will make it possible to assess genetic risks for hundreds of conditions with a single genetic test. In anticipation of these advances, there is a critical need to examine current genetic service delivery models, evaluate and assess these models, determine key components and develop new, efficient and effective models for providing genetic services for a growing and changing population of patients (Guttmacher et al. 2001; Battista et al. 2012; Hawkins and Hayden 2011). Defining and standardizing the classification of service delivery models (SDMs) will allow us to study the efficiency, efficacy, and impact of various methods of service delivery on access to genetic counselors. This, in turn, will help genetic counselors adapt their practices accordingly. The use of common terminology will be important to our profession and to other health care providers in understanding and describing these models of genetic counseling service delivery.

In 2009, the Institutes of Medicine (IOM) held a roundtable on Innovations in Service Delivery in the Age of Genomics and acknowledged the growing strain that predictive and new genetic testing place on an already strained system along with the need for improved characterization of the current service delivery system (IOM 2009). Additionally, incorporation of genetic counseling services into primary health care has become a priority identified by the Health and Human Services Secretary's Advisory Committee on Genetics, Health, and Society (Tuckson 2006). This committee recommended that policies be developed to allow genetic counselors and other genetic service providers to be included in coordinated care. SACGHS called for further guidance to ensure full access to genetic counseling services for all Americans, including identifying which health professionals are qualified to provide genetic counseling services and guidance on how to achieve full access.
To address access and workforce issues, innovative changes are being implemented by genetics professionals. The IOM workshop summary highlights new models for service delivery including telephone genetic consultation, web-based genetic counseling and testing (direct to consumer or physician involved), and a web-based genetic pharmacology service (IOM 2009). The use of group genetic counseling sessions, though not a new concept, has been reported as an effective method to provide genetic counseling in a research setting, however this result has not been analyzed in a clinical setting (Calzone et al. 2005; Ridge et al. 2009).

Additionally, over the past ten years, there have been tools developed to complement and enhance genetic counseling delivery such as videos, CD-ROMs, computer programs, slide presentations, websites and brochures (Dabney and Huelsman 2000; Green et al. 2005; Wang et al. 2005)). Other electronic family history tools and pedigree drawing programs (e.g. Progeny) have also been introduced to improve efficiency in collecting, storing and managing data. These tools may be able to improve efficiency in genetic counseling service delivery and their very development adds evidence to the rising demand for efficiency. But are these tools examples of service delivery models? With this paper we propose that these tools are not defining a SDM as much as enhancing the use of models themselves.

As the SDMTF began to dissect and define the goals and plan for the task force, it quickly became evident in the discussions that there was a lack of consistency of the understanding and usage of the terminology "service delivery models". Some members considered SDMs in terms of the requirements for physician supervision or billing capability while others considered SDMs in terms of how a patient receives information (in-person or telephone, for example). Still others considered counseling techniques utilized within the session as types of service delivery models. These varied interpretations of the term "service delivery model"and the components that may influence the success or challenges of a particular model highlighted the need to define SDMs for further discussion as well as for use in future study. The ability to study the effects of changes to genetic counseling service delivery as well as impact of new tools is limited by the lack of a standardized definition of a "Service Delivery Model". Therefore, the NSGC SDMTF proposes the following framework for classification of SDMs, shown in Fig. 1 and as described below.

\section{Defining Service Delivery Models}

Although the SDMTF did not set out to systematically review the literature for publication of SDMs, an informal literature review by several members was done to research 


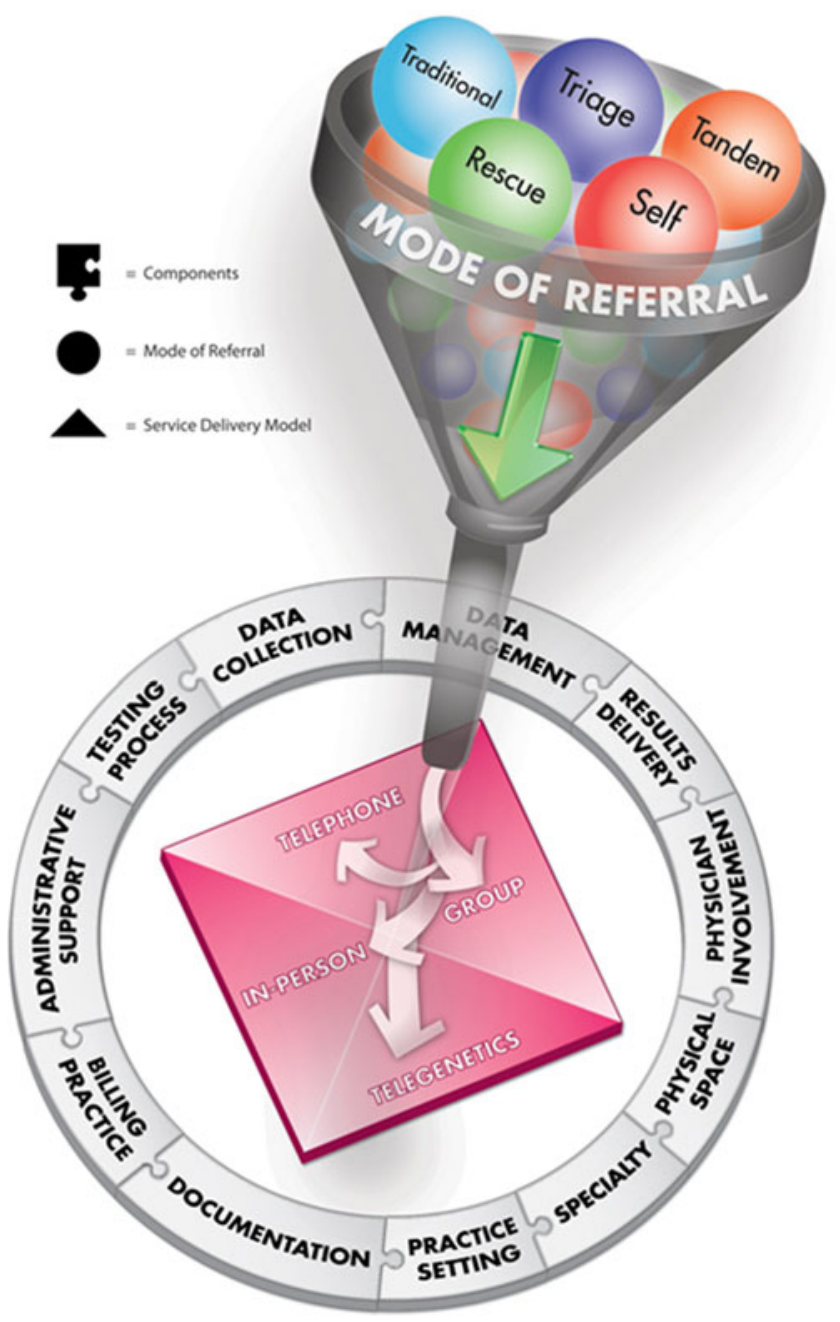

Fig. 1 Factors that influence genetic counseling service delivery models

terminology used with regard to service delivery in genetic counseling. We also considered how SDMs are defined in other areas of healthcare. The study of healthcare service delivery in primary care and healthcare in general appears to be variable in the literature. SDMs are often measured in the context of how a specific service is accessed, how a patient is determined to be eligible for the service, where and how the service is delivered, and how ongoing patient care is provided (Hall et al. 2008; Shengelia et al. 2005). Through conversations among SDMTF members over the course of a year and input from genetic counselors in the field using innovative methods of service delivery, a set of language was agreed upon by the SDMFT to begin to address the charges laid out in the NSGC strategic plan. We present here for consideration the definintion of models of service delivery as the method in which genetic counseling services are delivered by genetic counselors. Service delivery models should be differentiated from components of service, which may alter how efficient or accessible the model may be in different situations, such as the tools discussed above (videos, computer programs, etc.), which would be examples of components. We also considered modes of referral to describe how patients access the service. This clarification allowed us to propose definitions for four basic models of service delivery that appear to be in current use in practice in the United States.

1) In-Person genetic counseling. This model reflects the historical model of providing service in health care. Patients are seen in-person, usually at a healthcare facility or private office. Many prior publications refer to this method of delivery as "face-to-face" genetic counseling (Gattas et al. 2001; Coelho et al. 2005; Zilliacus et al. 2011; Peshkin et al. 2008; Wham et al. 2010). While "face-to-face" describes the model well, we propose that "in-person" more accurately differentiates it from telemedicine counseling, which is provided face-to-face, but not in-person. There are several examples of the term "in-person" in use in the literature (Jenkins et al. 2007; Bradbury et al. 2011; Doughty Rice et al. 2010). Based on unpublished data from a 2010 SDMTF survey of the NSGC membership, the majority of genetic counseling is currently provided by the inperson model.

2) Telephone genetic counseling. Genetic counseling is delivered by telephone to patients. Telephone genetic counseling should be considered a model of service delivery when a patient is provided genetic counseling for a new indication or concern and the session is completed entirely via telephone. For example, an individual may receive telephone genetic counseling for preconception evaluation of family history and/or ethnic carrier screening. It parallels the in-person model of genetic counseling because it uses a highly trained professional in a one-on-one session. Patients seem to accept this model of delivery as well as an in-person model (Sutphen et al. 2010). We propose that delivering results by phone is not a model of service delivery, but rather a component of service delivery that could occur as a part of any of the models of service delivery.

3) Group genetic counseling. Multiple individuals are seen for genetic counseling together, usually for a common indication (e.g. advanced maternal age, positive quad screen or increased risk for hereditary breast cancer). The group typically includes patients who are unrelated to each other, but could include multiple family members who are there each for their own purposes, being seen for the same indication (e.g. siblings at-risk for hereditary breast cancer). Brief individual consults after the group session may or may not occur, and are considered additional components of the model. Another variable component of the delivery might 
include use of a video. This model was probably first used in the prenatal genetic counseling setting, although earlier publications on "group genetic counseling" were often referring to what we would consider support groups for hereditary conditions or pregnancy termination. (Heimler 1990; Young et al. 1986; Kaiser et al. 2002). There are more recent documented uses of group genetic counseling in the cancer genetics setting that report it is well-accepted by patients (Ridge et al. 2009; Calzone et al. 2005).

4) Telegenetics (web-based and telemedicine genetic counseling). Genetic counseling is provided remotely via video-conference or web-link, including visual and audio access. Telegenetics is well-received and shown to be comparable to in-person genetic counseling in terms of patient satisfaction and knowledge in many studies dating back a decade (Coelho et al. 2005; Gattas et al. 2001; Zilliacus et al. 2011; Meropol et al. 2011; Hooper et al. 2011).

\section{Defining Components of Service Delivery}

Limiting the discussion to the four service delivery models described allows us to begin to describe current practices. However, these four models do not capture the differences that exist in application of the models across institutions or specialties. For example, in-person genetic counseling may occur with or without a physician present. A physical exam is not always performed. Billing practices differ; some genetic counseling services are billed with a facility fee, some with a professional fee and some do not bill at all. The SDMTF proposes definitions for multiple components of service delivery that could potentially affect the delivery model's efficiency and accessibility (Table 1). The components of a SDM are likely interdependent; for example billing practices may be dependent on physician involvement requirements and physician involvement may be dependent on the billing practices. Additionally, not all of these components will be relevant to every SDM. Some will be more heavily weighed than others, and they are all dynamic. To attempt to convey this fluidity, we have chosen to represent these components as interconnecting puzzleshaped pieces in a wheel around the method of delivery (Fig. 1). It should be noted that this list includes key components of a SDM identified to date and will likely be expanded with further evaluation of SDMs.

Variable components of a model may be dependent on the clinical setting (university vs. public health), specialty (cancer vs. prenatal), insurance coverage and contracts, and availability of support staff. Different factors will impact the components of genetic service delivery, and each center will have limitations on the flexibility of these components.
Recognizing which components of service delivery can be altered to improve efficiency and accessibility will improve genetic services.

\section{Defining Modes of Referral}

When the SDMTF began discussions about service delivery models, the discussions included different routes in which patients access genetic counseling. Rather than having infinite models of delivery based on these subtle differences, we propose to define modes of referral as the entry point into any given service delivery model. The mechanism by which a patient accesses genetic counseling may be unique or innovative, but the service itself can still be categorized by one of the proposed models of delivery. The following were chosen based on discussion among the SDMTF members that seemed to best describe the processes of referral that most commonly occur as a starting point for discussion. A patient may be referred to any of the above models as an independent visit or as part of a multi-disciplinary clinic by one of these routes:

1) Traditional: A physician or other health-care provider recognizes an indication and refers for genetic counseling. Example: An obstetrician refers a pregnant woman with a family history of muscular dystrophy to a genetic counselor.

2) Tandem: A collaborative relationship in which the initial genetic counseling is provided by another health care professional. All patients are then referred to a genetic counselor for follow up. Example: A pediatrician provides the initial counseling for developmental delay and orders chromosome microarray analysis (CMA). The patient is referred to a genetics counselor to review the results and consider further work-up.

3) Triage: A collaborative relationship in which the service is provided by another health care professional. Select patients are then referred to a genetic counselor as needed based on complexity. Example: An oncologist orders BRCA1/2 testing on a woman with early onset breast cancer. More complex cases, such as those with a significant family history of multiple types of cancers, are referred to a genetic counselor.

4) Rescue: Healthcare providers refer select patients to genetic counselors in the absence of a collaborative relationship and after they have encountered difficulty. Example: A primary care physician orders genetic testing for Huntington disease for a patient who has a family history of the condition. When the results are positive for Huntington disease, the patient is referred for genetic counseling because the physician is unsure what to tell the patient. 
Table 1 Components of service delivery

Data collection- Describes the method and timing of medical and/or family history collection. For example, this information may be collected prior to an appointment via mail, telephone, internet or collected at the time of the appointment.

Method of results delivery- Involves if and how results will be reported to a patient. For example, results may be discussed in a follow-up in-person appointment, by telephone or web-based method.

Physician involvement - A physician may or may not be present for some or all of the session. There are varying explanations for physician involvement, such as billing requirements, physical exam or management recommendations.

Billing practice- The method of billing, if done at all, will vary depending on the institution, setting, credentialing and licensure requirements.

Physical space- This may be a shared clinic or office space. There may be more than one place in which service is delivered, and it may be remote. Multi-disciplinary settings may be included in this component.

Location/Practice Setting - This describes where the service is located and can vary significantly. The service may be within a university, community hospital or public health setting, for example. This may also include satellite clinics.

Specialty - Describes the area of genetic specialty, including but not limited to prenatal, pediatrics, cancer, cardiology and adult.

Documentation- Practices may vary in many ways, such as separate or shared letters to patient and referring physicians, with or without documentation in a paper shadow chart and/or the use of electronic medical records.

Data management - This may include the use of pedigree drawing software, electronic medical records and other computer soft-ware to manage data (such as cancer genetics risk models, for example).

Mechanism of testing process - Outlines the methods used and details which staff members complete test requisition forms and carry out other tasks such as insurance pre-authorization for genetic tests.

Administrative support -Describes the personnel used to perform administrative tasks, such as patient scheduling.

5) Self Referred: Referral initiated by a patient. Example: A patient seeks out and contacts a genetic counselor for a family history of colon cancer because another relative was recently diagnosed.

\section{Discussion}

Clarifying the language of service delivery models in genetic counseling, limiting the discussion to models versus components of the model, and identifying modes by which patients access the models (referral patterns) will allow for a better understanding of the delivery of genetic counseling services. This common set of definitions will serve as a reference point for discussion about service delivery models, implementation of changes to improve service delivery, and development of research questions to evaluate efficiency. Expansion of these definitions is anticipated as more research is published and new models become more established. We expect the landscape of genetic counseling service delivery will continue to evolve over time. We propose that these definitions adequately describe current variations in service delivery and provide a starting point for additional study. As an example, a novel model described in the literature, the Collaborative model (Cohen et al. 2009), uses genetic counselors to support nongenetics health-care professionals in managing straightforward risk assessment and testing, while the genetic counselor provides direct patient care to those with a complex family history. Using the standard definitions proposed here, this service delivery model is an in-person model with a triage mode of referral, rather than a separate service delivery model.
Essentially, the interplay of the components of service delivery drive the selection and success of a model of service delivery, and to some degree the model may be circularly defined by these components (Fig. 1). However, it is notable that multiple models may work well in any one setting. Additional models may exist and are likely to develop over time. As new models are identified and defined, the list should be expanded to include them. Additionally, if some methods of delivery are used frequently enough with particular modes of referral and components of delivery, they may eventually earn the classification as a standalone service delivery model.

To keep pace with demand and improved technology, genetics service delivery must fully integrate into healthcare and find ways to optimally use information technology for patient education, testing and consultation. Genetic services need to improve efficiency, with attention to quality and ethical considerations. The variable impact of the components in each service delivery model may be measured concretely in different ways, such as wait time to access the service, how many patients a genetic counselor can see, how long is spent per patient in delivering the service, and how well a service is reimbursed. Comparing these measures may provide concrete data about how the components influence the efficiency and accessibility of genetic counseling services, as well as their influence on reimbursement, and is an important area for further study. Actually defining efficiency, accessibility and outcomes measures is beyond the scope of this paper, but hopefully these definitions will provide a framework to discuss SDMs and begin to outline studies to evaluate these very important topics. 
Quality and success of a service delivery model are key determinants in the selection thereof. As these are dependent on multiple factors specific to the institution or individual genetic counselor, these are not directly addressed in this paper. However, the evaluation of success and quality will require assessment of the impact of the components on service delivery and vice versa from the perspectives of the genetic counselor, institution, third party payer, and patient. By providing the terminology to evaluate individual components, these definitions provide the foundation for future research into the success and quality of various service delivery models in different settings.

It is notable when considering new delivery models that healthcare providers and consumers may not have the same ideals for optimal service provision. Valued attributes of genetic counseling shared by patients and providers are described as those that are local and accessible, family oriented, include regular follow up, a listening and empathetic relationship and time to talk (McAllister et al. 2008). These need to be weighed with the need for efficiency and cost-containment. Future assessment of service delivery models should include these values. These definitions proposed in this paper will promote clarity and discussion among genetic counseling as we address these issues.

\section{Conclusion}

This paper is the first to synthesize and offer definitions of genetic service delivery models, key components and modes of referral. In addition, we present the need for such clarity of terminology, which will effectively enable future research to address the efficiency and efficacy of genetic counseling services. There will be no "one-size-fits-all" service delivery model. As we identify different models, individual providers will likely modify these over time to be efficient, billable and accessible for their unique situation, with the underlying goal of maintaining quality.

New genetic counseling models must be efficient and financially viable, but at the same time maintain quality of care (Guttmacher et al. 2001). Common language to define service delivery models will help with the development of outcomes based studies to document the value of genetic services and develop best practice models. Ultimately, this will move us toward the full integration of genetics into clinical practice.

Acknowledgements We would like to acknowledge all the members of the SDMTF for their contributions to this work. We also would like to acknowledge graphic designer Gregory Nixon for his work to produce the SDM figure.

\section{References}

Battista, R. N., Blancquaert, I., Laberge, A. M., van Schendel, N., \& Leduc, N. (2012). Genetics in health care: an overview of current and emerging models. Public Health Genomics, 15(1), 34-45.

Bradbury, A. R., Patrick-Miller, L., Fetzer, D., Egleston, B., Cummings, S. A., Forman, A., et al. (2011). Genetic counselor opinions of, and experiences with telephone communication of BRCA1/2 test results. Clin Genet, 79(2), 125-131. doi:10.1111/j.13990004.2010.01540.x.

Calzone, K. A., Prindiville, S. A., Jourkiv, O., Jenkins, J., DeCarvalho, M., Wallerstedt, D. B., et al. (2005). Randomized comparison of group versus individual genetic education and counseling for familial breast and/or ovarian cancer. J Clin Oncol, 23(15), 3455-3464. doi:23/15/3455[pii]10.1200/JCO.2005.04.050.

Coelho, J. J., Arnold, A., Nayler, J., Tischkowitz, M., \& MacKay, J. (2005). An assessment of the efficacy of cancer genetic counselling using real-time videoconferencing technology (telemedicine) compared to face-to-face consultations. Eur J Cancer, 41(15), 2257-2261. doi:10.1016/j.ejca.2005.06.020.

Cohen, S. A., McIlvried, D., \& Schnieders, J. (2009). A collaborative approach to genetic testing: a community hospital's experience. $J$ Genet Couns, 18(6), 530-533. doi:10.1007/s10897-009-9243-y.

Dabney, M. K., \& Huelsman, K. (2000). Counseling by computer: breast cancer risk and genetic testing. Developed by the University of Wisconsin-Madison Department of Medicine and the Program in Medical Ethics. Genet Test, 4(1), 43-44. doi:10.1089/ 109065700316453.

Doughty Rice, C., Ruschman, J. G., Martin, L. J., Manders, J. B., \& Miller, E. (2010). Retrospective comparison of patient outcomes after in-person and telephone results disclosure counseling for BRCA1/2 genetic testing. Fam Cancer, 9(2), 203-212. doi:10.1007/s10689-009-9303-3.

Gattas, M. R., MacMillan, J. C., Meinecke, I., Loane, M., \& Wootton, R. (2001). Telemedicine and clinical genetics: establishing a successful service. Journal of Telemedicine and Telecare, 7(suppl 2), 68-70. doi:10.1258/1357633011937191.

Green, M. J., Peterson, S. K., Baker, M. W., Friedman, L. C., Harper, G. R., Rubinstein, W. S., et al. (2005). Use of an educational computer program before genetic counseling for breast cancer susceptibility: effects on duration and content of counseling sessions. Genet Med, 7(4), 221-229.

Guttmacher, A. E., Jenkins, J., \& Uhlmann, W. R. (2001). Genomic medicine: who will practice it? A call to open arms. Am J Med Genet, 106(3), 216-222. doi:10.1002/ajmg.10008.

Hall, A. G., Lemak, C. H., Steingraber, H., \& Schaffer, S. (2008). Expanding the definition of access: it isn't just about health insurance. J Health Care Poor Underserved, 19(2), 625-638. doi:10.1353/hpu.0.0011.

Hawkins, A. K., \& Hayden, M. R. (2011). A grand challenge: providing benefits of clinical genetics to those in need. Genet Med, 13(3), 197200. doi:10.1097/GIM.0b013e31820c056e.

Heimler, A. (1990). Group counseling for couples who have terminated a pregnancy following prenatal diagnosis. Birth Defects Original Article Series, 26(3), 161-167.

Hooper, B., Buckman, M., \& Edwards, M. (2011). Evaluation of satisfaction of parents with the use of videoconferencing for a pediatric genetic consultation. Twin Res Hum Genet, 14(4), 343-346.

IOM (Institute of Medicine) (2009). Innovations in service delivery in the age of genomics: Workshop summary. Washington, DC: The National Academies Press. http://www.nap.edu/catalog/ 12601.html.

Jenkins, J., Calzone, K. A., Dimond, E., Liewehr, D. J., Steinberg, S. M., Jourkiv, O., et al. (2007). Randomized comparison of phone versus in-person $B R C A 1 / 2$ predisposition genetic test result 
disclosure counseling. Genet Med, 9(8), 487-495. doi:10.1097/ GIM.0b013e31812e6220.

Kaiser, A. S., Ferris, L. E., Pastuszak, A. L., Llewellyn-Thomas, H., Johnson, J.-A., Conacher, S., et al. (2002). The effects of prenatal group genetic counselling on knowledge, anxiety and decisional conflict: issues for nuchal translucency screening. Journal of Obstetrics \& Gynaecology, 22(3), 246-255. doi:10.1080/01443610220130508.

McAllister, M., Payne, K., Macleod, R., Nicholls, S., Donnai, D., \& Davies, L. (2008). What process attributes of clinical genetics services could maximise patient benefits? Eur J Hum Genet, 16 (12), 1467-1476. doi:10.1038/ejhg.2008.121.

Meropol, N. J., Daly, M. B., Vig, H. S., Manion, F. J., Manne, S. L., Mazar, C., et al. (2011). Delivery of Internet-based cancer genetic counselling services to patients' homes: a feasibility study. Journal of Telemedicine and Telecare, 17(1), 36-40. doi:10.1258/jtt.2010.100116.

Peshkin, B. N., Demarco, T. A., Graves, K. D., Brown, K., Nusbaum, R. H., Moglia, D., et al. (2008). Telephone genetic counseling for high-risk women undergoing BRCA1 and BRCA2 testing: rationale and development of a randomized controlled trial. Genet Test, 12(1), 37-52. doi:10.1089/gte.2006.0525.

Ridge, Y., Panabaker, K., McCullum, M., Portigal-Todd, C., Scott, J., \& McGillivray, B. (2009). Evaluation of group genetic counseling for hereditary breast and ovarian cancer. J Genet Couns, 18(1), 87-100. doi:10.1007/s10897-008-9189-5.

Shengelia, B., Tandon, A., Adams, O. B., \& Murray, C. J. L. (2005). Access, utilization, quality, and effective coverage: an integrated conceptual framework and measurement strategy. Social Science \& Medicine, 61(1), 97-109. doi:10.1016/j.socscimed.2004.11.055.

Sutphen, R., Davila, B., Shappell, H., Holtje, T., Vadaparampil, S., Friedman, S., et al. (2010). Real world experience with cancer genetic counseling via telephone. Familial Cancer, 9(4), 681689. doi:10.1007/s10689-010-9369-y.

Tuckson, R. (2006). Coverage and Reimbursement of Genetic Tests and Services: Report of the Secretary's Advisory Committee on Genetics, Health, and Society. http://oba.od.nih.gov/oba/sacghs/ reports/CR report.pdf.

Wang, C., Gonzalez, R., Milliron, K. J., Strecher, V. J., \& Merajver, S. D. (2005). Genetic counseling for BRCA1/2: a randomized controlled trial of two strategies to facilitate the education and counseling process. Am J Med Genet A, 134A(1), 66-73. doi:10.1002/ajmg.a.30577.

Wham, D., Vu, T., Chan-Smutko, G., Kobelka, C., Urbauer, D., \& Heald, B. (2010). Assessment of clinical practices among cancer genetic counselors. Fam Cancer. doi:10.1007/s10689-010-9326-9.

Young, R., Jorgenson, R., \& Shapiro, S. (1986). Efficacy of and patient preference for three counseling formats. Journal of Craniofacial Genetics and Developmental Biology, 6, 3-14.

Zilliacus, E. M., Meiser, B., Lobb, E. A., Kelly, P. J., Barlow-Stewart, K., Kirk, J. A., et al. (2011). Are videoconferenced consultations as effective as face-to-face consultations for hereditary breast and ovarian cancer genetic counseling? Genet Med, 13(11), 933-941. doi:10.1097/GIM.0b013e3182217a19. 